\title{
Dynamic Hubbard model for solids with hydrogen-like atoms
}

\author{
J. E. Hirsch \\ Department of Physics, University of California, San Diego \\ La Jolla, CA 92093-0319
}

\begin{abstract}
We discuss how to construct a tight binding model Hamiltonan for the simplest possible solid, composed of hydrogen-like atoms. A single orbital per atom is not sufficient because the on-site electron-electron repulsion mixes in higher energy orbitals. The essential physics is captured by a dynamic Hubbard model with one electronic orbital and an auxiliary spin degree of freedom per site. We point out that this physics can lead to a substantial shift in the position and width of electronic energy bands relative to what is predicted by conventional band structure calculations.
\end{abstract}

PACS numbers:

\section{INTRODUCTION}

Just like understanding the physics of the simplest atom, hydrogen, proved essential to the understanding of more complex atoms, we argue that understanding the essential physics of a solid composed of hydrogenlike atoms is a prerequisite to understand more complex solids. When such a simplified solid is discussed it is usually to illustrate the physics of the Mott metal-insulator transition occurring for a $1 / 2$-filled band 1 ]. Here instead we focus on the physics of energy bands with band filling larger than $1 / 2$, where there are necessarily some atomic orbitals / Wannier states that are doubly occupied by electrons.

Tight binding Hamiltonians commonly used to model correlated electrons in solids such as the Hubbard model, the Anderson model, the $t-J$ model and the Holstein model fail to describe the essential physical fact that double occupancy of an atomic orbital changes the wavefunction of the single electron in the orbital [2]. In fact, the wavefunction for the two electrons is not even a single Slater determinant. An approximate description with a single Slater determinant can be given, but the single electron orbital in the two-electron wavefunction is different from that in the single electron wavefunction, due to electron-electron repulsion. This is of course well known in atomic physics since at least the work of Hartree 85 years ago [3, 4], but surprisingly has not yet found its way into the mainstream description of interacting electrons in solids [5].

In tight binding models with one orbital per site the singly occupied orbital in an atom is denoted by

$$
\left|\uparrow>=c_{\uparrow}^{\dagger}\right| 0>
$$

and the doubly occupied orbital by

$$
\left|\uparrow \downarrow>=c_{\uparrow}^{\dagger} c_{\downarrow}^{\dagger}\right| 0>
$$

which in particular implies that

$$
<\uparrow\left|c_{\uparrow}^{\dagger}\right| 0>=<\downarrow\left|c_{\uparrow}\right| \uparrow \downarrow>=1
$$

and implies electron-hole symmetry at the atomic level, i.e. creating an electron in the empty orbital (left side of Eq. (2)) is the same as creating a hole in the doubly occupied orbital (right side of Eq. (2)). This is assumed to be the case in all the conventional Hamiltonians mentioned above. However it is qualitatively incorrect because the doubly occupied state is never a single Slater determinant but rather a linear combination of Slater determinants involving higher single electron states [6] :

$$
\begin{gathered}
\left|\uparrow \downarrow>=\sum_{m, n} A_{m n} c_{m \uparrow}^{\dagger} c_{n \downarrow}^{\dagger}\right| 0> \\
\sum_{m, n}\left|A_{m n}\right|^{2}=1
\end{gathered}
$$

where the sum runs over a complete set of atomic orbitals, with the lowest single particle orbital denoted by $m=0$, i.e. $c_{0 \sigma}=c_{\sigma}$, as well as over continuum states [7]. Hence

$$
c_{\uparrow}\left|\uparrow \downarrow>=\sum_{n} A_{0 n} c_{n \downarrow}^{\dagger}\right| 0>=A_{00}\left|\downarrow>+\sum_{n \neq 0} A_{0 n} c_{n \downarrow}^{\dagger}\right| 0>
$$

and

$$
<\downarrow\left|c_{\uparrow}\right| \uparrow \downarrow>=A_{00} \neq 1
$$

contrary to Eq. (2). For the particular case $Z=2$, the numerical value of $A_{00}$, calculated in ref. [7], is 0.9624 . Both the fact that $A_{00}<1$ and the fact that there is more than one term on the right side of Eq. (4a) has important consequences for the properties of solids formed by these atoms [6]. Fundamentally, the fact that Eq. (2) is invalid and Eq. (4) is valid reflects the basic physical fact that atoms are not electron-hole symmetric, nor are solids.

While this physics is ubiquitous, it is important to analyze when it will be important and when it can be neglected, just as other ubiquitous physics like spinorbit interaction, electron-phonon interaction, Hund's rule terms, longer-range Coulomb interactions etc are neglected in the single band Hubbard model because their effect is quantitatively small and/or because it is assumed their effects don't change the physics qualitatively. The energy eigenvalues for an electron in the single-particle orbitals of hydrogen-like atoms are $\epsilon_{n}=-13.6\left(Z^{2} / n^{2}\right) e V$, with $Z$ the charge of the nucleus. 
When we apply this model to more complicated atoms, $Z$ will be the net charge of the atom when there are 0 electrons in the orbital under consideration, and $Z-1$, $Z-2$ the net charges of the atom when there are one and two electrons in the orbital respectively. The coefficients $A_{m n}$ in Eq. (3) can be expressed as

$$
A_{m n}=\frac{<\uparrow \downarrow\left|H_{I} c_{m \uparrow}^{\dagger} c_{n \downarrow}^{\dagger}\right| 0>}{E_{0}-\left(\epsilon_{m}+\epsilon_{n}\right)}
$$

with $H_{I}=e^{2} /\left|\vec{r}-\vec{r}^{\prime}\right|$ (in first quantized form) the electron-electron interaction and $E_{0}$ the exact ground state energy of the doubly occupied orbital. The matrix elements of $H_{I}$ in the numerator of Eq. (5) are of order $Z$, while the energy differences in the denominator are of order $Z^{2}$ for $(m, n) \neq(0,0)$, so only in the limit of large $Z$ will one have $A_{m n} \sim 0$ for $(m, n) \neq(0,0), A_{00} \sim 1$ and $E_{0} \sim 2 \epsilon_{1}+U$. As $Z$ decreases and the electron-ion interaction becomes comparable to the electron-electron interaction $(Z \sim 1)$ the matrix elements $A_{m n}$ become appreciable which implies through the sum rule Eq. (3b) that $A_{00}$ becomes increasingly smaller.

Put another way: using the conventional Hubbard model for the description of the doubly-occupied atomic states amounts to doing first order perturbation theory in the interaction $H_{I}$ for the eigenvalue of the doubly occupied orbital and zeroth order perturbation theory for the eigenstate. This is absurd unless the spacing between non-interacting energy levels, which is of order $Z^{2}$, is much larger than the matrix elements between noninteracting states and the perturbation, which is of order $Z$ in general (provided it is not zero because of different angular momenta). This is never the case in real atoms, and the effects discussed here will be largest for negatively charged ions (small $Z$ ), where the mixing between the lowest energy states and higher energy states due to electron-electron repulsion will be larger because of the smaller spacing between energy states and hence $A_{00}$ will be smaller.

In addition to $Z$ being small, another requirement for this physics to be important in the solid state is that the band described by this Hamiltonian be more than half full, so that doubly-occupied atomic states are unavoidable. For the band less than half-full, the conventional Hubbard $U$ will keep the amplitude of doubly-occupied sites small and this physics will not be very important.

In recent years, 'dynamic Hubbard models' have been proposed to incorporate this physics in the simplest possible way [6, 8 15]. Models that have been discussed so far contain either a single atomic orbital per site plus an auxiliary boson (spin 1/2 [9, 13] or harmonic oscillator [10, 12] ) degree of freedom, or two orbitals per site and no auxiliary boson [11, 14]. They reflect the fundamental fact that creating an electron in the empty atomic orbital is qualitatively different from creating a hole in the doubly occupied orbital for the reasons exposed above.

One consequence of this physics is that electrical conduction in an almost full band is qualitatively different from electrical conduction in an almost empty band
[6, 15], and in particular we have shown that this can lead to pairing and superconductivity in electronic energy bands that are almost full, driven by lowering of electronic kinetic energy [16, 17]. We have also recently shown that this leads to a tendency of the system to expel electrons from the interior to the surface when electronic energy bands are almost full [18, 19].

In this paper we discuss the modeling of a solid with hydrogen-like atoms with a dynamic Hubbard model with a spin degree of freedom. Extension to other types of atoms is briefly considered. In particular, we find that the physics described by the dynamic Hubbard model will lead to a shift in the position and width of energy bands that are full or almost full relative to what is predicted by conventional band structure calculations.

\section{THE HYDROGEN-LIKE ATOM}

The simplest non-trivial form for the ground state wave function of two electrons in a hydrogen-like atom is of the Hartree form

$$
\begin{gathered}
\Psi\left(r_{1}, r_{2}\right)=\varphi_{\tilde{Z}}\left(r_{1}\right) \varphi_{\tilde{Z}}\left(r_{2}\right) \\
\varphi_{\tilde{Z}}\left(r_{1}\right)=\sqrt{\frac{\tilde{Z}^{3}}{\pi}} e^{-\tilde{Z}_{r} / a_{0}}
\end{gathered}
$$

with $a_{0}=\hbar^{2} /\left(m_{e} e^{2}\right)$ the Bohr radius. This is an approximation to the linear combination of Slater determinants given by Eq. (3). For the singly occupied orbital the wavefunction is Eq. (6b) with $\tilde{Z}=Z$, with $Z$ e the nuclear charge. For the doubly occupied orbital the kinetic and potential energies for each electron and the electronelsctron interaction energy are given by

$$
\begin{gathered}
E_{k i n}(\tilde{Z})=\frac{\hbar^{2}}{2 m_{e} a_{0}^{2}} \tilde{Z}^{2}=\frac{e^{2}}{2 a_{0}} \tilde{Z}^{2} \\
E_{\text {pot }}(\tilde{Z})=-\frac{e^{2}}{a_{0}} Z \tilde{Z} \\
E_{e e}(\tilde{Z})=\frac{5}{8} \frac{e^{2}}{a_{0}} \tilde{Z}
\end{gathered}
$$

so that the total energy for the two-electron ion is

$$
E_{t o t}=\frac{e^{2}}{a_{0}}\left(\tilde{Z}^{2}-2 Z \tilde{Z}+\frac{5}{8} \tilde{Z}\right)
$$

and is minimized by

$$
\tilde{Z}=Z-\frac{5}{16} \equiv Z-\delta \equiv \bar{Z}
$$

so that the wavefunction expands upon double occupancy from radius $a_{0} / Z$ to radius $a_{0} / \bar{Z}$. The spatial extent of 


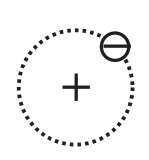

$\mathrm{E}(1)$

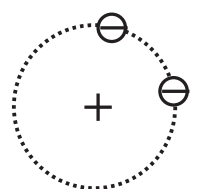

$\mathrm{E}(2)$

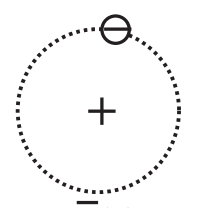

$\overline{\mathrm{E}}(1)$

(c)

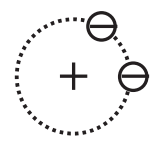

$\overline{\mathrm{E}}(2)$

(d)
FIG. 1: States (a) and (b) are the lowest energy states for 1 and 2 electrons, with unexpanded and expanded orbital respectively. States (c) and (d) are one and two-electron states with expanded and unexpanded orbital respectively, and correspondingly higher energies. In the conventional Hubbard model only states (a) and (d) are considered.

the wavefunction becomes larger the smaller the ionic charge $Z$, and diverges as $Z \rightarrow 5 / 16=0.3125$.

Figure 1 shows schematically the four states of interest here. (a) and (b) are the ground states of the atom with one and two-electrons respectively, with unexpanded and expanded orbits respectively and energies

$$
\begin{aligned}
E(1)= & E_{k i n}(Z)+E_{p o t}(Z)=-\frac{e^{2}}{2 a_{0}} Z^{2} \\
E(2) & =2\left(E_{k i n}(\bar{Z})+E_{\text {pot }}(\bar{Z})\right)+E_{e e}(\bar{Z}) \\
& =-\frac{e^{2}}{a_{0}}\left(Z^{2}-2 \delta Z+\delta^{2}\right)
\end{aligned}
$$

The states (c) and (d) are excited states (but they are not orthogonal to the ground states): (c) for the single electron in the expanded orbit, and (d) for the two electrons in the non-expanded orbit, with energies

$$
\begin{gathered}
\bar{E}(1)=E_{k i n}(\bar{Z})+E_{p o t}(\bar{Z})=-\frac{e^{2}}{2 a_{0}}\left(Z^{2}-\delta^{2}\right) \\
\bar{E}(2)=2\left(E_{k i n}(Z)+E_{p o t}(Z)\right)+E_{e e}(Z) \\
=-\frac{e^{2}}{a_{0}} Z(Z-2 \delta)
\end{gathered}
$$

respectively. We note that the energies satisfy

$$
\begin{aligned}
& \bar{E}(1)-E(1)=\frac{\delta^{2}}{2} \frac{e^{2}}{a_{0}} \\
& \bar{E}(2)-E(2)=\delta^{2} \frac{e^{2}}{a_{0}}
\end{aligned}
$$

so the difference in the energy of the excited state and the ground state is independent of $Z$, and in addition they satisfy

$$
\bar{E}(2)-E(2)=2(\bar{E}(1)-E(1)) .
$$

The right side of Eq. (13) is twice the cost in single electron energy in expanding the orbital from its single electron radius to the larger radius apropriate to the twoelectron atom. The two-electron atom pays that cost but gains twice as much from the reduction in electronelectron repulsion achieved by expanding the orbital:

$$
E_{e e}(Z)-E_{e e}(\bar{Z})=2 \delta^{2} \frac{e^{2}}{a_{0}}=4(\bar{E}(1)-E(1)) .
$$

From this point of view it can be said that the orbital expansion is driven by lowering of the electron-electron interaction energy at a cost of single-particle energy.

It is also interesting to ask whether the expansion is 'kinetic energy driven' or 'potential energy driven'. Upon double occupancy and resulting orbital expansion the electron-ion potential energy increases more than the reduction in electron-electron repulsion, giving rise to a net increase in potential energy:

$$
\begin{aligned}
\bar{E}_{p o t}-E_{p o t} & =2\left(E_{p o t}(\bar{Z})-E_{p o t}(Z)\right)+E_{e e}(\bar{Z})-E_{e e}(Z) \\
& =2 \delta \frac{e^{2}}{a_{0}}(Z-\delta)
\end{aligned}
$$

which is always positive due to the requirement that $\bar{Z}>$ 0 . On the other hand the kinetic energy always decreases

$$
\bar{E}_{k i n}-E_{k i n}=2\left(E_{k i n}(\bar{Z})-E_{k i n}(Z)\right)=-2 \delta \frac{e^{2}}{a_{0}}\left(Z-\frac{\delta}{2}\right)
$$

for a total energy lowering

$$
\bar{E}_{t o t}-E_{t o t}=E(2)-\bar{E}(2)=-\delta^{2} \frac{e^{2}}{a_{0}}
$$

so one can say that the orbital expansion upon double occupancy is always "kinetic energy driven". Also, in comparing the lowering of kinetic energy Eq. (16) with the lowering of electron-electron repulsion energy Eq. (14) we find that the former is larger as long as

$$
Z>\frac{3}{2} \delta
$$

or $\bar{Z}>\delta / 2=0.15625$, a rather small value. The ratio of kinetic energy lowering to electron-electron energy lowering is

$$
\frac{\Delta E_{k i n}}{\Delta E_{e e}}=\frac{2 Z}{\delta}-\frac{1}{2}
$$

so that for hydrogen for example $(Z=1)$ the lowering of kinetic energy upon double occupancy and resulting orbital expansion is more than three times larger than the lowering of electron-electron repulsion energy.

In the conventional Hubbard model the orbital expansion is not considered. The value of the Hubbard $U$ in the unrelaxed atomic orbital is

$$
U_{\text {bare }}=E_{e e}(Z)=\frac{5}{8} \frac{e^{2}}{a_{0}} Z
$$




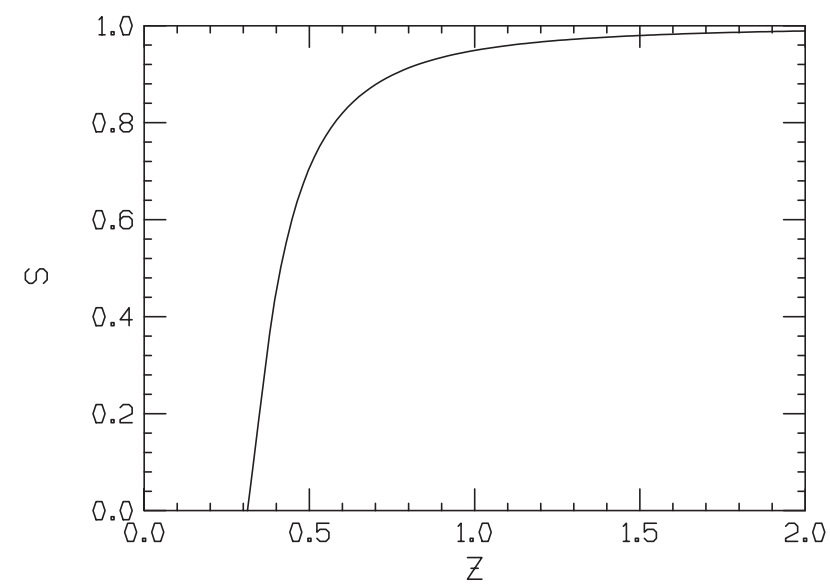

FIG. 2: Overlap matrix element of the expanded and unexpanded orbital $S$ versus atomic charge $Z$

In the expanded orbital the electron-electron repulsion is lower, given by

$$
U_{e x p}=E_{e e}(\bar{Z})=\frac{5}{8} \frac{e^{2}}{a_{0}}(Z-\delta)
$$

However the actual "effective $U$ " is larger than $U_{\text {exp }}$, since the single particle energy cost in expanding the orbital has to be taken into account. The effective $U$ is given by

$$
U_{\text {eff }}=E(2)-2 E(1)=\frac{5}{8} \frac{e^{2}}{a_{0}}\left(Z-\frac{\delta}{2}\right)
$$

so that it is the average of the repulsions in the expanded and unexpanded orbitals. Note that $U_{\text {bare }}$ and $U_{\text {exp }}$ are also given by the expressions

$$
\begin{aligned}
& U_{\text {bare }}=\bar{E}(2)-2 E(1) \\
& U_{\text {exp }}=E(2)-2 \bar{E}(1) .
\end{aligned}
$$

Finally, it is of interest to compute the overlap matrix element of the single particle expanded and unexpanded orbital

$$
S=<\varphi_{Z} \mid \varphi_{\bar{Z}}>=\frac{(Z \bar{Z})^{3 / 2}}{\left(\frac{Z+\bar{Z}}{2}\right)^{3}}=\frac{\left(1-\frac{\delta}{Z}\right)^{3 / 2}}{\left(1-\frac{\delta}{2 Z}\right)^{3}} .
$$

This is plotted in Fig. 2 as function of the ionic charge $Z$. The overlap decreases as the ionic charge decreases and approaches zero for $Z \rightarrow 5 / 16$ where the expanded orbital becomes infinitely large. Note however that the Hartree approximation underestimates the magnitude of these effects. For example, for $Z=2$ the Hartree wave function gives $S=0.9892$ and the highly accurate Hylleraas wave function that includes radial and angular correlations and should be essentially exact gives $S=0.9810$. This value is obtained from the square root of the overlap matrix element of the two-electron wave function with the wave function of two electrons in the unexpanded orbitals.

The matrix element $S$ plays a key role in determining the properties of electrons in the energy band generated by this atomic orbital [12]: the quasiparticle weight decreases and the effective mass of the carriers increases as the band filling increases, with the magnitude of these effects determined by the deviation of $S$ from unity.

\section{DYNAMIC HUBBARD MODEL WITH AUXILIARY SPIN DEGREE OF FREEDOM}

\section{A. Simplest model}

The simplest form for the site Hamiltonian for a dynamic Hubbard model with an auxiliary spin 1/2 degree of freedom is 13 ]

$$
\begin{gathered}
H_{i}=\epsilon_{e}+\epsilon_{0}\left(n_{i \uparrow}+n_{i \downarrow}\right)+\omega_{0} \sigma_{x}^{i}+g \omega_{0} \sigma_{z}^{i} \\
+\left(U_{0}-2 g \omega_{0} \sigma_{z}^{i}\right) n_{i \uparrow} n_{i \downarrow} \\
\epsilon_{e}=\omega_{0} \sqrt{1+g^{2}}
\end{gathered}
$$

so that the (lowest) energy is zero when there are no electrons at the site. The spin part of the Hamiltonian is

$$
\begin{array}{r}
H_{\text {spin }}(n=0, n=1)=\omega_{0} \sigma_{x}^{i}+g \omega_{0} \sigma_{z}^{i} \\
H_{\text {spin }}(n=2)=\omega_{0} \sigma_{x}^{i}-g \omega_{0} \sigma_{z}^{i}
\end{array}
$$

when there are $n$ electrons at the site. The eigenvalues of these spin Hamiltonians are

$$
\epsilon= \pm \omega_{0} \sqrt{1+g^{2}}
$$

and the eigenvectors

$$
\begin{aligned}
& |n>=u(n)|+>+v(n) \mid-> \\
& |\bar{n}>=v(n)|+>-u(n) \mid->
\end{aligned}
$$

with $\mid n>$ denoting the ground state and $\mid \bar{n}>$ the excited state for the spin with $n$ electrons at the site, $\mid+>$ and $\mid->$ the basis states for the spin degree of freedom, and

$$
\begin{gathered}
u(n) \equiv u=\sqrt{\frac{1}{2}\left(1-\frac{g}{\sqrt{1+g^{2}}}\right)} \\
v(n) \equiv v=-\sqrt{\frac{1}{2}\left(1+\frac{g}{\sqrt{1+g^{2}}}\right)} .
\end{gathered}
$$

for $n=0,1$ and

$$
u(2)=v(1)
$$




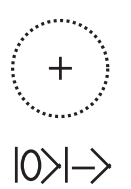

$\mathrm{E}(0)$
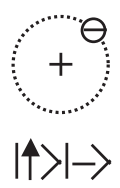

$\mathrm{E}(1)$

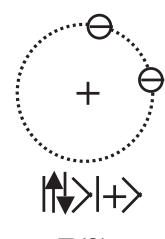

$\mathrm{E}(2)$

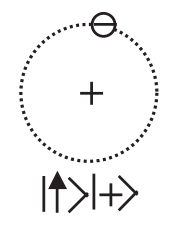

$\overline{\mathrm{E}}(1)$

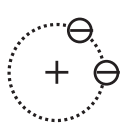

$|A|>\mid>$

$\overline{\mathrm{E}}(2)$

(e)
FIG. 3: Dynamic Hubbard model representation of the atomic states. The $\mid->$ and $\mid+>$ spin states correspond to the unexpanded and expanded orbital in the limit $g \rightarrow \infty$. For finite $g$, they are replaced by the states $\mid 1>$ and $\mid 2>$ respectively (see text).

$$
v(2)=u(1)
$$

This Hamiltonian is particularly simple because the eigenvalues Eq. (27) are independent of the site occupation and the eigenvectors for different occupations are related in the simple way given by Eq. (30), and for this reason it was chosen in Ref. [13]. Later in this section we consider other possible versions of this model where these expressions take a less simple form.

The essential physics of this (and the other versions of this) Hamiltonian is that the lowest state of the spin is the same for 0 or 1 electron at the site, and different for 2 electrons at the site. For large $g$, the lowest energy states are approximately $\mid->$ for $n=0$ and $n=1$ and $\mid+>$ for $n=2$. The change in the state of the spin represents the orbital expansion as shown schematically in Fig. 3. The overlap matrix elements between small and large orbitals Eq. (24) is thus given by

$$
S=<1|2>=| 2 u v \mid=\frac{1}{\sqrt{1+g^{2}}}
$$

so that $g$ is given by

$$
g=\sqrt{\frac{1}{S^{2}}-1}
$$

The lowest site energies in the dynamic Hubbard model Eq. (25) are

$$
\begin{gathered}
E(0)=0 \\
E(1)=\epsilon_{0} \\
E(2)=2 \epsilon_{0}+U_{0}
\end{gathered}
$$

If we destroy an electron from the ground state of the two-electron atom the spin remains in state $\mid 2>$. The expectation value of the Hamiltonian for one electron with the spin state $\mid 2>$ then gives the energy of one electron in the expanded orbital, $\bar{E}(1)$ :

$$
\bar{E}(1)=<2|H(n=1)| 2>=\epsilon_{0}+\omega_{0} \sqrt{1+g^{2}}+\frac{g^{2}-1}{\sqrt{1+g^{2}}} \omega_{0}
$$

and similarly the expectation value of the two-electron Hamiltonian with the spin state $\mid 1>$ gives the energy of the two-electron atom with the unexpanded orbital:

$$
\begin{aligned}
\bar{E}(2) & =<1|H(n=2)| 1>=2 \epsilon_{0}+U_{0} \\
& +\omega_{0} \sqrt{1+g^{2}}+\frac{g^{2}-1}{\sqrt{1+g^{2}}} \omega_{0}
\end{aligned}
$$

Note however that this implies

$$
\bar{E}(2)-E(2)=\bar{E}(1)-E(1)
$$

which disagrees with Eq. (13) obeyed by the atomic energies. Thus, it is not possible to find parameters in the model Eq. (25) that will match the four energies of the atomic states considered. So we ignore the energy $\bar{E}(2)$ and determine the parameters in the model by equating the energies $E(1), E(2)$ and $\bar{E}(1)$ to the atomic energies and by Eq. (32). The result is

$$
\begin{gathered}
\omega_{0}=\frac{25 S}{1024\left(1-S^{2}\right)} \frac{e^{2}}{a_{0}} \\
\epsilon_{0}=-\frac{e^{2}}{2 a_{0}} Z^{2} \\
U_{0}=U_{\text {eff }}=\frac{5}{8} \frac{e^{2}}{a_{0}}\left(Z-\frac{5}{32}\right)
\end{gathered}
$$

Thus, Eqs. (32) and (37) determine the parameters in the dynamic Hubbard model Hamiltonian as function of the atomic parameters.

Let us consider some numerical examples. (a) For $Z=$ $1, S=0.949$ and the parameters in the model are $g=$ $0.332, \omega_{0}=6.34 \mathrm{eV}, \epsilon_{0}=-13.6 \mathrm{eV}, U_{0}=14.35 \mathrm{eV}$. (b) For $Z=0.3557, S=0.5$ and the parameters in the model are $g=1.732, \omega_{0}=0.443 \mathrm{eV}, \epsilon_{0}=-1.72 \mathrm{eV}$, $U_{0}=3.39 \mathrm{eV}$.

\section{B. Alternative form of the model}

We can generalize the model Eq. (25) to

$$
\begin{aligned}
H_{i}= & \epsilon_{e}+\epsilon_{0}\left(n_{i \uparrow}+n_{i \downarrow}\right)+\omega_{0} \sigma_{x}^{i}+g \omega_{0} \sigma_{z}^{i} \\
& +\left(U_{0}-a g \omega_{0} \sigma_{z}^{i}\right) n_{i \uparrow} n_{i \downarrow} .
\end{aligned}
$$

with $\epsilon_{e}$ is still given by Eq. (25b), so that $E(0)=0$. As long as the parameter $a>1$ the physics is essentially the same as that of Eq. (25) (which is the case $a=2$ ), particularly for large $g$ : the state of the spin changes drastically when a hole is created at the doubly occupied site but doesn't change when an electron is created at the empty site. The eigenvalues of the spin Hamiltonian are now

$$
\epsilon= \pm \omega_{0} \sqrt{1+g^{2}}
$$


for 0 or 1 electron at the site, and

$$
\epsilon= \pm \omega_{0} \sqrt{1+(a-1)^{2} g^{2}}
$$

for 2 electrons at the site, so the eigenvalues are no longer independent of site occupation for any $a \neq 2$.

Introducing the parameter $a$ allows us to satisfy the condition Eq. (13). It will be satisfied if the relation

$$
\sqrt{1+(a-1)^{2} g^{2}}=2 \sqrt{1+g^{2}}
$$

holds, hence if $a$ is given by

$$
a=a(g)=1+\sqrt{4+\frac{3}{g^{2}}} .
$$

$a$ increases monotonically as $g$ decreases, from $a=3$ for $g \rightarrow \infty$ to $a(g) \sim \sqrt{3} / g$ for $g \rightarrow 0$. The energies are given by

$$
\begin{gathered}
E(1)=\epsilon_{0} \\
\bar{E}(1)=\epsilon_{0}+\frac{1+2 g^{2}+g \sqrt{3+4 g^{2}}}{2 \sqrt{1+g^{2}}} \omega_{0} \\
E(2)=2 \epsilon_{0}+U_{0}-\omega_{0} \sqrt{1+g^{2}} \\
\bar{E}(2)=2 \epsilon_{0}+U_{0}+\frac{g^{2}+g \sqrt{3+4 g^{2}}}{\sqrt{1+g^{2}}} \omega_{0}
\end{gathered}
$$

so that the atomic relation $\bar{E}(2)-E(2))=2(\bar{E}(1)-E(1))$ holds for all $g$. In the limit of large $g, a=3$ and we have $\bar{E}(1)=\epsilon_{0}+2 g \omega_{0}, E(2)=2 \epsilon_{0}+U_{0}-g \omega_{0}, \bar{E}(2)=$ $2 \epsilon_{0}+U_{0}+3 g \omega_{0}$. For any $g$ the eigenvector amplitudes are given by

$$
\begin{gathered}
u(2)=\sqrt{\frac{1}{2}\left(1+\frac{\sqrt{3+4 g^{2}}}{2 \sqrt{1+g^{2}}}\right)} \\
v(2)=-\sqrt{\frac{1}{2}\left(1-\frac{\sqrt{3+4 g^{2}}}{2 \sqrt{1+g^{2}}}\right)}
\end{gathered}
$$

The coupling constant $g$ is again determined from the condition $S=<1 \mid 2>$ which now is

$$
S=\frac{\sqrt{3+2 g^{2}-g \sqrt{3+4 g^{2}}}}{2 \sqrt{1+g^{2}}}
$$

with $S$ given by Eq. (24). It can be seen from Eq. (44) that as $g \rightarrow 0, S \rightarrow \sqrt{3} / 2=0.866$. In that limit the spin part of the Hamiltonian is

$$
H_{i}^{s p i n}(n=1)=\omega_{0} \sigma_{x}^{i}
$$

$$
H_{i}^{s p i n}(n=2)=\omega_{0} \sigma_{x}^{i}-\sqrt{3} \omega_{0} \sigma_{z}^{i}
$$

with energies $\epsilon(1)= \pm \omega_{0}$ and $\epsilon(2)= \pm 2 \omega_{0}$ giving rise to $<1 \mid 2>=\sqrt{3} / 2$ and satisfying the condition Eq. (13).

Therefore, we conclude that the Hamiltonian of the form Eq. (38) with $a$ chosen to satisfy the condition Eq. (13) can only be used to match the 4 atomic energies Eqs. (10) and (11) provided $S<0.866$, which from Eq. (24) corresponds to $Z<0.673$. In any event the regime of the model that gives rise to interesting properties in the solid is large $g$, corresponding to small $S$ and $Z$.

So to find the appropriate parameters in the model for a given $Z<0.673$ and corresponding $S$ given by Eq. (24) we first find $g$ from Eq. (44). $\epsilon_{e}$ and $\epsilon_{0}$ are still given by Eqs. (25b) and (37b), and the other parameters are given by

$$
\begin{aligned}
& \omega_{0}=\frac{25}{256} \frac{e^{2}}{a_{0}} \frac{\sqrt{1+g^{2}}}{1+2 g^{2}+\sqrt{3 g^{2}+4 g^{4}}} \\
& U_{0}=\frac{5}{8} \frac{e^{2}}{a_{0}}\left(Z-\frac{5}{32}\right)+\omega_{0} \sqrt{1+g^{2}} .
\end{aligned}
$$

For the numerical example given in the previous section $Z=0.3557, S=0.5$, we obtain for the parameters in this model $g=2 / \sqrt{3}=1.1554, \omega_{0}=25 e^{2} /\left(256 \sqrt{21} a_{0}\right)=$ $0.580 \mathrm{eV}, \epsilon_{0}=-1.72 \mathrm{eV}, U_{0}=4.28 \mathrm{eV}$, versus $g=1.732$, $\omega_{0}=0.443 \mathrm{eV}, \epsilon_{0}=-1.72 \mathrm{eV}, U_{0}=3.39 \mathrm{eV}$ in the simpler model Eq. (25).

\section{Third version of the model}

Finally we consider also the Hamiltonian Eq. (38) with $a=3$ independent of the value of $g$. Recall that this value of $a$ satisfies the atomic condition Eq. (13) only for $g \rightarrow$ $\infty$. However, the deviation from this condition is much smaller than for the model of the form Eq. (25). The lowest eigenvalues for different occupations are $E(1)=\epsilon_{0}$ and

$$
E(2)=2 \epsilon_{0}+U_{0}+\omega_{0} \sqrt{1+g^{2}}-\omega_{0} \sqrt{1+4 g^{2}}
$$

and the energies for one electron in the expanded orbital and two electrons in the unexpanded orbital are respectively

$$
\begin{array}{r}
\bar{E}(1)=\epsilon_{0}+\omega_{0} \sqrt{1+g^{2}}+\frac{2 g^{2}-1}{\sqrt{1+4 g^{2}}} \omega_{0} \\
\bar{E}(2)=2 \epsilon_{0}+U_{0}+\frac{3 g^{2}}{\sqrt{1+g^{2}}} \omega_{0}
\end{array}
$$

The relation Eq. (13) is no longer satisfied, rather

$$
\bar{E}(2)-E(2)=C(\bar{E}(1)-E(1))
$$




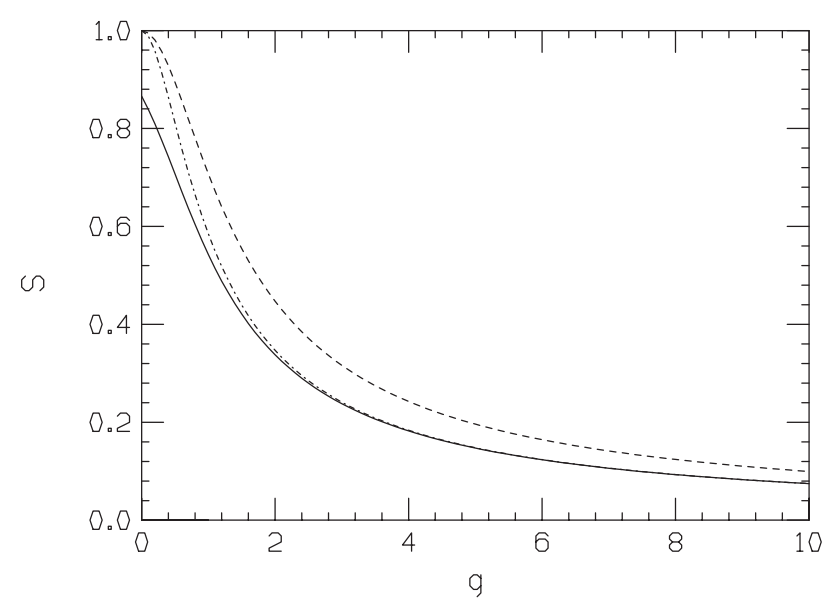

FIG. 4: Overlap matrix element $S$ versus coupling constant $g$ for the first (Eq. 25, dashed line), second (Eq. 38, solid line) and third (Eq. 38 with $a=3$, dash-dotted line) version of the model

$$
C=\sqrt{\frac{1+4 g^{2}}{1+g^{2}}}
$$

so for example $C=1.96,1.92,1.84,1.58$ for $g=4,3,2,1$, closer to the atomic value $C=2$ than in the model Eq. (25) for which $C=1$ for all $g$.

The eigenvectors are given by Eq. (29) for $n=0,1$, and for $n=2$ by

$$
\begin{gathered}
u(2)=\sqrt{\frac{1}{2}\left(1+\frac{2 g}{\sqrt{1+4 g^{2}}}\right)} \\
v(2)=-\sqrt{\frac{1}{2}\left(1-\frac{2 g}{\sqrt{1+4 g^{2}}}\right)},
\end{gathered}
$$

The coupling constant $g$ is determined from the condition $S=<1 \mid 2>$ which is now

$$
S=\sqrt{\frac{1}{2}\left(1+\frac{1-2 g^{2}}{\sqrt{1+5 g^{2}+4 g^{4}}}\right)}
$$

and the other parameters in the Hamiltonian are given by Eq. (37b) and

$$
\begin{gathered}
\omega_{0}=\frac{25}{512} \frac{e^{2}}{a_{0}} \frac{\sqrt{1+4 g^{2}}}{2 g^{2}-1+\sqrt{\left(1+g^{2}\right)\left(1+4 g^{2}\right)}} \\
U_{0}=\frac{5}{8} \frac{e^{2}}{a_{0}}\left(Z-\frac{5}{32}\right)+\omega_{0} \sqrt{1+4 g^{2}}-\omega_{0} \sqrt{1+g^{2}} .
\end{gathered}
$$

Figure 4 shows the overlap matrix element $S$ versus coupling constant $g$, fig. 5 shows the ratio of energy differences $C$ (Eq. (49)) and fig. 6 shows the coupling constant $g$ versus atomic charge $Z$ for the three versions of the model. The second and third version become equivalent in the strong coupling regime which corresponds to the case of negatively charged ions (small $Z$ ).

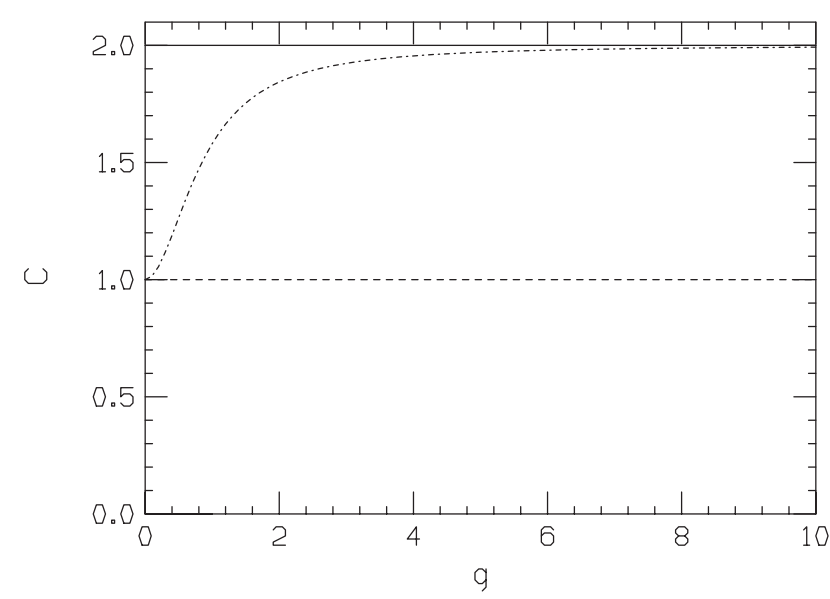

FIG. 5: Ratio of energies $C=(\bar{E}(2)-E(2)) /(\bar{E}(1)-E(1))$ versus coupling constant $g$ for the first (Eq. 25, dashed line), second (Eq. 38, solid line) and third (Eq. 38 with $a=3$, dash-dotted line) version of the model. In the atom, $C=2$ for all values of the coupling constant.

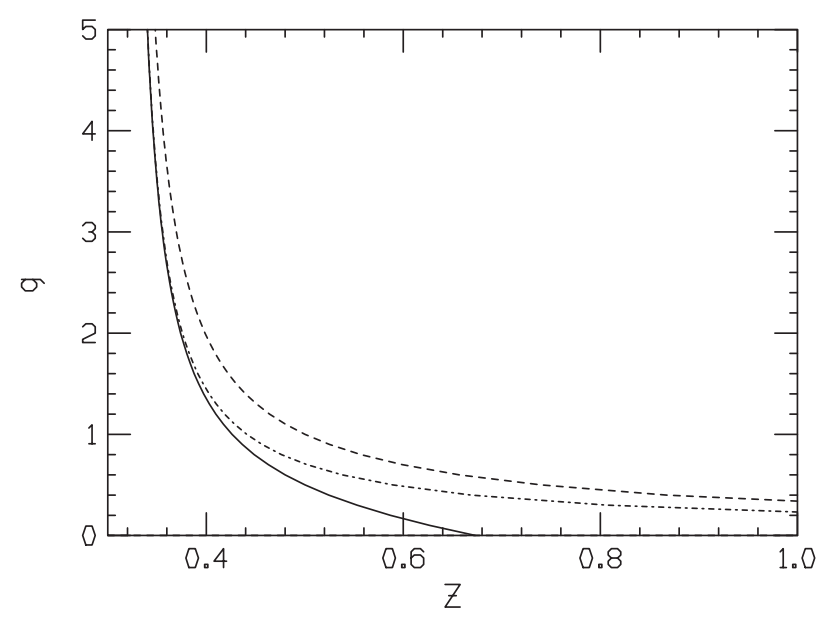

FIG. 6: Coupling constant $g$ versus atomic charge $Z$ for the first (Eq. 25, dashed line), second (Eq. 38, solid line) and third (Eq. 38 with $a=3$, dash-dotted line) version of the model

\section{EFFECTIVE HAMILTONIAN}

The Hamiltonian for the lattice system is

$$
H=\sum_{i} H_{i}-t \sum_{i, j}\left(c_{i \sigma}^{\dagger} c_{j \sigma}+h . c .\right)
$$

acting on the product Hilbert space of electron states and spin states. We denote the site states by the product of electron and spin states. The action of a fermion creation operator is

$$
\begin{array}{r}
c_{i \uparrow}^{\dagger}|0>| 0>=|\uparrow>| 0>=|\uparrow>| 1> \\
c_{i \uparrow}^{\dagger}|\downarrow>| 1>=S|\uparrow \downarrow>| 2>+\bar{S}|\uparrow \downarrow>| \overline{2}>
\end{array}
$$


with $S=<1|2>, \bar{S}=<1| \overline{2}>. \mid \overline{2}>$ denotes the higher energy spin state for two electrons at the site:

$$
|\overline{2}>=v(2)|+>-u(2) \mid->.
$$

The first term in Eq. (54b) describes the ground-state to ground-state (diagonal) transition, i.e. the process where the orbital expands when doubly occupied. The second term is the process where the doubly occupied site ends up in an excited state. We define quasiparticle fermion operators $\tilde{c}_{i \sigma}^{\dagger}$ by the relation

$$
c_{i \sigma}^{\dagger}=\left[1-(1-S) \tilde{n}_{i,-\sigma}\right] \tilde{c}_{i \sigma}^{\dagger}+\bar{S}|\uparrow \downarrow>| \overline{2}><1|<\sigma|
$$

to describe the diagonal transitions and to obtain the effective low energy Hamiltonian we ignore the second term in Eq. (56):

$$
\begin{aligned}
& H_{e f f}=\sum_{i j} t_{i j}^{\sigma}\left(\tilde{c}_{i \sigma}^{\dagger} \tilde{c}_{j \sigma}+h . c .\right) \\
&+U_{\text {eff }} \sum_{i} \tilde{n}_{i \uparrow} \tilde{n}_{i \downarrow}+\epsilon_{0} \sum_{i}\left(\tilde{n}_{i \uparrow}+\tilde{n}_{i \downarrow}\right) \\
& t_{i j}^{\sigma}=t\left[1-(1-S) \tilde{n}_{i,-\sigma}\right]\left[1-(1-S) \tilde{n}_{i-\sigma}\right]
\end{aligned}
$$

to describe the low-energy physics.

As discussed elsewhere [16], this Hamiltonian gives rise to high temperature superconductivity when the band is almost full and the overlap matrix element $S$ is sufficiently small. The condition for superconductivity for an almost full band is 20]

$$
S \leq \sqrt{1-\frac{U_{e f f}}{D}}
$$

with $D$ the unrenormalized bandwidth $(D=2 z t$, with $z$ the number of nearest neighbors to a site). Note that as the net ionic charge $Z$ decreases the overlap matrix element $S$ decreases (Fig. 2) and in addition $U_{\text {eff }}$ (Eq. (22)) decreases, so that the condition Eq. (65) is more easily satisfied. Thus according to this model high temperature superconductivity is favored by having negatively charged ions.

The factor $S^{2}$ represents the quasiparticle weight when the band is almost full [12]. The second term in Eq. (54b) gives rise to incoherent processes that contribute to the high energy optical absorption and photoemission spectra for bands that are almost full [6]. Thus, high temperature superconductivity in this model is associated with small quasiparticle weight and large spectral weight for incoherent processes $\left(S^{2}+\bar{S}^{2}=1\right)$, physical features found in high $T_{c}$ cuprates [21 23].

\section{SHIFT OF ENERGY BANDS}

We argue that the orbital relaxation effect described by the dynamic Hubbard model will shift the location of

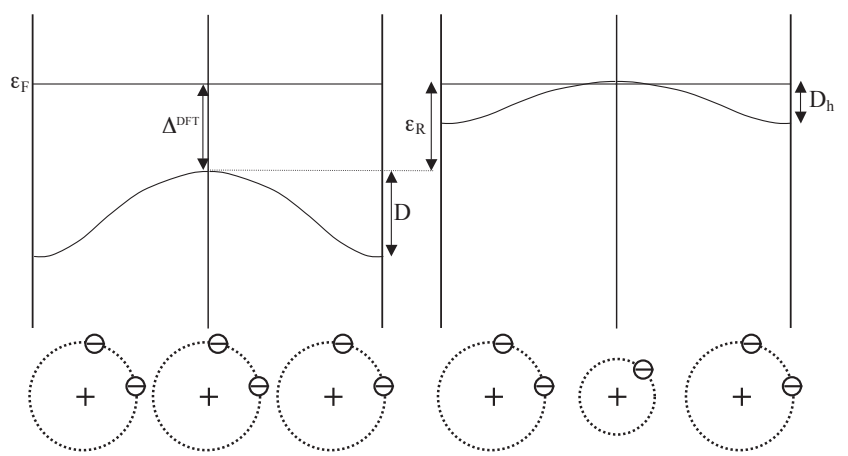

(a) Density functional calculation

(b) Dynamic Hubbard calculation

FIG. 7: In a standard band structure calculation using density functional theory (a), the orbital relaxation energy when an electron is removed from the band is not taken into account. In a correct calculation that includes the physics described by the dynamic Hubbard model (b), the top of the band shifts upward by the relaxation energy Eq. (60b) and the band narrows as given by Eq. (62).

energy bands that are almost full relative to what would be predicted by density functional band structure calculations.

Consider a band structure calculation within density functional theory (DFT) that predicts a full band several $\mathrm{eV}$ below the Fermi energy $\epsilon_{F}$, as shown in Fig. 7. The calculation assumes that each atomic orbital is doubly occupied, and predicts that the Fermi level has to come down a distance $\Delta^{D F T}$ for doped holes to go into that band, hence that doped holes will go into other bands that cross the Fermi energy. The distance between the top of the band and the Fermi energy is

$$
\Delta^{D F T}=E_{\text {final }}^{D F T}-E_{\text {initial }}
$$

where $E_{\text {initial }}$ and $E_{\text {final }}^{D F T}$ are the initial and final energies of the system upon bringing an electron from the top of the band to the Fermi energy.

However, the DFT calculation does not take into account the local relaxation of the orbital when an electron is removed from this band, since on the average all orbitals remain doubly occupied for infinitesimal hole doping. This has been pointed out particularly by Fulde and coworkers 24 26] as a fundamental limitation of band structure calculations within the local density approximation in density functional theory. Instead, a correct calculation that includes the orbital relaxation physics described by the dynamic Hubbard model Eq. (53) or its effective low energy Hamiltonian Eq. (57) would yield as final energy

$$
E_{\text {final }}^{D H}=E_{\text {final }}^{D F T}-\epsilon_{R}
$$

with

$$
\epsilon_{R}=\bar{E}(1)-E(1)
$$

the orbital relaxation energy discussed in the previous 
sections. Hence

$$
\Delta^{D H}=\Delta^{D F T}-\epsilon_{R}
$$

may become negative as shown on the right panel in Fig. 7, indicating that doped holes will go into this band, contrary to the predictions of density functional theory.

In addition to the shift in the position of the energy band, density functional calculations also will miss the fact that the orbital relaxation will narrow the band [11] due to the reduced overlap matrix element between expanded and unexpanded orbital. When the band is almost full the hopping amplitude from Eq. (57b) is $t_{h}=S^{2} t$ and hence the bandwidth is

$$
D_{h}=S^{2} D
$$

as shown schematically in Fig. 7. The fact that band structure calculations miss this band narrowing effect has also been emphasized recently by Casula and coworkers 27.

For hydrogen-like atoms within the Hartree approximation the orbital relaxation energy is given by Eq. (12a) which yields $\epsilon_{R}=1.33 \mathrm{eV}$. Note however that we can also obtain the orbital relaxation energy from

$$
\epsilon_{R}=\frac{1}{2}\left(U_{\text {bare }}-U_{\text {eff }}\right)
$$

from Eqs. (23) and (13). For real hydrogen-like ions we can obtain $U_{\text {eff }}$ from the difference of appropriate ionization energies and obtain [8]

$$
U_{\text {bare }}-U_{\text {eff }}=4.15 \mathrm{eV}
$$

within $10 \%$ for $Z$ between 1 and 8 (U $U_{\text {bare }}-$ $U_{\text {eff }}=4.15 \mathrm{eV}, 4.10 \mathrm{eV}, 4.22 \mathrm{eV}, 4.22 \mathrm{eV}, 4.21 \mathrm{eV}, 4.19 \mathrm{eV}$, $4.15 \mathrm{eV}, 4.05 \mathrm{eV}$ for $Z=1,2, \ldots 8)$. Therefore,

$$
\epsilon_{R} \sim 2.1 \mathrm{eV}
$$

for hydrogen-like ions, about $60 \%$ higher than in the Hartree approximation.

For negatively charged oxygen ions as in the $\mathrm{Cu}-\mathrm{O}$ planes of high $T_{c}$ superconductors the relaxation energy is likely to be even larger. We can estimate [28] the bare $U$ from the Slater integral [2] $F^{o}(2 p, 2 p) \sim 20.5 \mathrm{eV}$ for $O^{\circ}$ (the spherically averaged Coulomb repulsion between two electrons in the $p$ shell) and the effective $U$ from the electron affinities of $O_{o}$ and $O^{-}$

$$
\begin{aligned}
& E\left(O^{-}\right)-E(O)=-1.45 \mathrm{eV} \\
& E\left(O^{2-}\right)-E\left(O^{-}\right)=8.75 \mathrm{eV}
\end{aligned}
$$

yielding

$$
U_{\text {eff }}=E\left(O^{2-}\right)+E(O)-2 E\left(O^{-}\right)=10.2 \mathrm{eV}
$$

and hence for the relaxation energy

$$
\epsilon_{R}=\frac{1}{2}\left(U_{\text {bare }}-U_{\text {eff }}\right)=5.1 \mathrm{eV}
$$

Band structure calculations using density functional theory predict that in high $T_{c}$ cuprates the band resulting from overlap of oxygen $p_{\pi}$ orbitals in the plane oriented perpendicular to the $\mathrm{Cu}-\mathrm{O}$ bond is several $\mathrm{eV}$ below the Fermi energy [29]. Instead, we have argued that to explain high temperature superconductivity in the cuprates it is necessary that doped holes occupy the oxygen $p_{\pi}$ band rather than the $\mathrm{Cu}-O p_{\sigma}$ band usually assumed [16, 30]. From the arguments presented here we conclude that the orbital relaxation energy will shift the $O p_{\pi}$ band several $\mathrm{eV}$ upward, hence it is plausible to conclude that doped holes will occupy that band, contrary to the band structure calculation predictions that don't take orbital relaxation energy into account [31].

\section{SUMMARY AND DISCUSSION}

We have argued that the conventional Hubbard model that ignores the fact that atomic orbitals expand with increasing electron occupation misses important physics of real materials, that a simple extension of the Hubbard model that we call "dynamic Hubbard model" takes into account. We have analyzed in detail a dynamic Hubbard model with a single atomic orbital and an auxiliary spin $1 / 2$ degree of freedom. Other possible realizations of dynamic Hubbard models are a purely electronic model with two orbitals per site and a single orbital model with an auxiliary harmonic oscillator degree of freedom [6]. The effects described by dynamic Hubbard models will be quantitatively more important for bands arising from conduction through negatively charged ions.

We considered the energies of the various states involved in hydrogen-like atoms, and how to model these atoms with various alternative forms of a dynamic Hubbard Hamiltonian with an auxiliary spin $1 / 2$ degree of freedom. The effective Hamiltonian resulting from the different versions of the model is the same as far as the low energy degrees of freedom are concerned, with different mappings of the Hamiltonian parameters to the atomic parameters.

Finally, we have argued that conventional band structure calculations miss two important properties of electronic energy bands for cases where the bands are full or almost full that are described by dynamic Hubbard models: (1) the position of the band is higher and (2) the width of the band is smaller than predicted by band structure calculations.

Thus, we argue that to learn about the properties of the simplest possible solid composed of hydrogen-like atoms, when there is more than one electron per atom (band more than half full), it is inappropriate both to use the conventional Hubbard model, conventional band structure calculations, or band structure calculations combined with dynamical mean field theory of the conventional Hubbard model 32]. Instead one should solve the dynamic Hubbard model Hamiltonian Eq. (53) with one of the versions of the site Hamiltonians $H_{i}$ discussed, 
for parameters in the model obtained by the mapping to the atomic parameters discussed in Sect. III, or alternatively the low energy effective Hamiltonian Eq. (57). Exact diagonalization methods [13], quantum Monte Carlo [33] or dynamical mean field theory 34] can be used to study these Hamiltonians. The physics that results, which in particular includes superconductivity when the band is almost full [16] and Eq. (58) is satisfied, is very different than the physics obtained by the conventional methods and we believe it reflects the physics of the real system, while the physics obtained from the conventional methods does not. Of course the same considerations apply to more complicated real materials.

We have proposed that this model could apply to the high $T_{c}$ cuprates provided doped holes go into a full oxygen $p_{\pi}$ band $[16,30]$. Conventional band structure calculations place this band several $\mathrm{eV}$ below the Fermi energy [29], however, we have shown here that the orbital relaxation effect described by dynamic Hubbard models will shift the position of full bands or nearly full bands upward by several $e V$. Hence this supports the possibility that doped holes in the high $T_{c}$ cuprates may occupy the $O p_{\pi}$ orbitals rather than the $O p_{\sigma}$ orbitals as usually assumed. A detailed analysis of this model for the high $T_{c}$ cuprates including the $C u-O p_{\sigma}$ band is given in [35].

Superconductivity through this mechanism is favored both by having a low $U_{\text {eff }}$ and by having a small $S$. Both $U_{\text {eff }}$ and $S$ become smaller when the effective ionic charge $Z$ becomes smaller (Eqs. (22) and (24)). While we have shown this explicitly here only for hydrogen-like ions, it is clear that it will also be the case in general. Thus, superconductivity is favored by having negatively charged ions. The fact that negative ions have smaller on-site Coulomb repulsion should also be favorable for superconductivity within other superconductivity mechanisms that rely on electron pairing. For example, within the conventional BCS-electron-phonon interaction mechanism $\mu^{*}$ should be smaller for systems with negative ions, leading to a higher $T_{c}$. It is surprising that this has not been pointed out before in the literature to our knowledge. This suggests for example that hole-doped $L i B C$ should have a substantially lower transition temperature than $M g B_{2}$ because in substituting $C$ for $B$ and $L i$ for $M g$, half of the ions in the planes where conduction occurs become $C^{o}$ rather than $B^{-}$, thus increasing $Z$ from 1 to 2 and as a consequence increasing the effective Coulomb repulsion on the $C$ sites. Instead, it has been predicted, based on electron-phonon calculations that presumably don't take this effect into account, that hole-doped $\mathrm{LiBC}$ should have a substantially higher transition temperature than $M g B_{2}$ [36].
[1] M. Imada, A. Fujimori and Y. Tokura, Rev. Mod. Phys. 70, 1039 (1998).

[2] J.C. Slater. "Quantum Theory of Atomic Structure", Mc Graw Hill, New York, 1960.

[3] D.R. Hartree, "The Wave Mechanics of an Atom with a Non-Coulomb Central Field. Part I. Theory and Methods", Math. Proc. of the Cambridge Philos. Soc. 24, 89110, 111-132, 426-437 (1928).

[4] D.R. Hartree, "The Calculation of Atomic Structures", John Wiley \& Sons, New York, 1957.

[5] J. E. Hirsch, Physica B 199\&200, 366 (1994).

[6] J. E. Hirsch, Phys. Rev. B 65, 184502 (2002).

[7] J. Hutchinson, M. Baker and F. Marsiglio, Europ. J. of Phys. 34, 111 (2013).

[8] J.E. Hirsch, Phys.Rev. Lett. 87, 206402 (2001).

[9] J.E. Hirsch, Phys. Lett. A 134, 451 (1989).

[10] J.E. Hirsch, Physica C 201: 347-361 (1992).

[11] J.E. Hirsch, Phys. Rev. B 43, 11400 (1991).

[12] J.E. Hirsch, Phys. Rev. B 6214487 and 14998 (2000).

[13] J.E. Hirsch, Phys. Rev. B 65, 214510 and 66, 064507 (2002).

[14] J.E. Hirsch, Rev. B 67, 035103 (2003).

[15] J.E. Hirsch, Physica C 364-365, 37 (2001).

[16] J.E. Hirsch and F. Marsiglio, Phys. Rev. B 39, 11515 (1989).

[17] J.E. Hirsch and F. Marsiglio, Phys. Rev. B 62,15131 (2000).

[18] J.E. Hirsch, Phys.Rev. B 87184506 (2013).
[19] J.E. Hirsch, Physica Scripta 88, 035704 (2013).

[20] J.E. Hirsch, Phys. Rev. 47, 5351 (1993).

[21] H. Ding et al, Phys. Rev. Lett. 87, 227001 (2001).

[22] K.M. Shen et al, Phys. Rev. Letters 93, 267002 (2004).

[23] N. Trivedi, Nature Physics 4, 163 (2008).

[24] P. Fulde, Int. J. Quantum Chem. 76, 385 (2000).

[25] A. Stoyanova, L. Hozoi, P. Fulde and H. Stoll, J. Chem. Phys. 131, 044119 (2009).

[26] L. Hozoi, U. Birkenheuer, P. Fulde, A. Mitrushchenkov, and H. Stoll , Phys. Rev. B 76, 085109 (2007).

[27] M. Casula, Ph. Werner, L. Vaugier, F. Aryasetiawan, T. Miyake, A. J. Millis, and S. Biermann, Phys. Rev. Lett. 109, 126408 (2012).

[28] J.E. Hirsch and S. Tang, Phys.Rev. B 40, 2179 (1989).

[29] W.E. Pickett, Rev. Mod. Phys. 61, 433 (1989).

[30] J.E. Hirsch and S. Tang, Sol.St. Comm. 69: 987(1989).

[31] This possibility was also proposed early on by Goddard and coworkers: Y. Guo, J.M. Langlois and W.A. Goddard, Science 239, 896 (1988).

[32] G. Kotliar et al, Rev. Mod. Phys. 78. 865 (2006).

[33] K. Bouadim, M. Enjalran, F. Hbert, G. G. Batrouni, and R. T. Scalettar, Phys. Rev. B77, 014516 (2008)

[34] G.H. Bach, J.E. Hirsch and F. Marsiglio,, Phys. Rev. B 82, 155122 (2010).

[35] J.E. Hirsch, arXiv:1407.0042 (2014).

[36] H. Rosner and W.E. Pickett, Phys. Rev. Lett. 88, 127001 (2002). 